\title{
Exploring inter-rater reliability and measurement properties of environmental ratings using kappa and colocation quotients
}

\author{
Jonas Björk ${ }^{1^{*}}$, Ralf Rittner ${ }^{1}$ and Ellen Cromley ${ }^{1,2}$
}

\begin{abstract}
Background: Available evidence suggest that perceptions or ratings of the neighborhood, e.g. as being green, walkable or noisy, are important for effects on health and wellbeing, also after controlling for objective measures of identical or similar features. When evaluating effects of the perceived environment, it is important that measurement properties and the reliability of the environmental ratings are evaluated before decisions about how these ratings should be handled in the statistical analyses are made. In this paper we broaden the usage of two association measures, the well-known kappa statistic and the novel colocation quotient (CLQ), to studies of inter-rater reliability and of associations between different categorical ratings in spatial contexts.

Methods: We conducted reliability analysis of a survey instrument for assessing perceived greenness at geographical point locations, here the close outdoor environment within 5-10 minutes walking distance from home. Data were obtained from a public health survey conducted in 2008 in Scania, southern Sweden ( $n=27$ 967 participants).

Results: The results demonstrate the usefulness of kappa and CLQ as tools for assessing reliability and measurement properties of environmental rating scales when used at geographical point locations. We further show that the two measures are interchangeable, i.e. kappa can be accurately approximated from CLQ and vice versa, but can be used for somewhat different purposes in reliability analyses. Inter-rater reliability between the nearest neighbors was demonstrated for all five items of the evaluated instrument for assessing perceived greenness, albeit with clear differences across the items.

Conclusion: Reliability analysis employing kappa and CLQ can be used as a basis for informed decisions about, for instance, how dichotomizations of the ratings should be defined and how missing or indefinite ratings should be handled. Such reliability analyses can thus serve as guidance for subsequent epidemiological studies of associations between environmental ratings, health and wellbeing.
\end{abstract}

Keywords: Epidemiologic methods, Spatial analysis, Reproducibility of results, Statistics as topic, Perceived greenness

\section{Background}

Perceptions and ratings of environmental attributes have been used extensively in studies of green environments and health [1-4], but also in studies of neighborhood resources for physical activity [5-7], traffic noise [8] and air quality $[9,10]$. There is evidence suggesting that perceptions of the neighborhood, e.g. as being green, walkable or

\footnotetext{
* Correspondence: jonas.bjork@med.lu.se

${ }^{1}$ Division of Occupational and Environmental Medicine, Lund University, SE-221 85 Lund, Sweden

Full list of author information is available at the end of the article
}

noisy, are important for effects on health and wellbeing, also after controlling for objective measures of identical or similar features $[1,6,8,11,12]$.

Comparisons of self-rated and objectively measured neighborhood attributes show associations (correlations) but generally low agreement [11,13-16]. The low agreement has been explained as a mismatch between perceptions and objective facts [11], but may also suggest that perceived and objective measures are capturing different aspects of the local environment [13]. However, the rating 
instruments may lack inter- or intra-rater reliability, thereby contributing to the low agreement with objectively measured attributes. Additionally, decisions about how to collapse or dichotomize categorical ratings, and how to handle indefinite or missing ratings, are often taken arbitrarily without an appropriate analysis of how the ratings are distributed and clustered spatially. Ignoring the spatial distribution of the ratings may add to the discrepancy between exposure measures based on perceptions and objective attributes.

Reliability of individual items in categorical assessment instruments is often evaluated by comparing observed with chance-expected agreement using the kappa statistic, either for a fixed set of raters [17] or for different sets of raters [18] for each assessed object or environment. Kappa is normally used as an overall measure of agreement for an entire rating instrument, but can also be calculated for the agreement between specific categories [18,19]. For ordinal scales, weights can be applied when calculating kappa to quantify the extent of the disagreement $[19,20]$.

The colocation quotient (CLQ) was introduced recently as a measure of spatial association among observations of a single categorical variable at point locations [21]. The CLQ builds on the concept of the location quotient, a widely used spatial measure of concentration in a location relative to a norm [22]. While the original purpose of the CLQ was to assess spatial associations between different categories (different classes of objects), it can also be used in analyses of spatial segregation, a phenomenon that occurs when members or ratings of the same category cluster spatially [23]. In the context of environmental ratings at point locations, clustering of identical or compatible ratings will be evidence of inter-rater agreement, i.e. that neighboring subjects agree on the quality of the environment they are rating. When such rating data are obtained from survey questions and the residential locations of the survey participants are known, the inter-rater agreement and reliability can be assessed by comparing the ratings of each participant with the first-order nearest neighbors. Calculated from a nearest neighbor contingency table [23], the CLQ will reflect the inter-rater reliability as a measure of how many times more likely than chance it is that the same categorical rating occurs among the neighbors. Kappa can also be calculated from the same table, providing an opportunity to compare kappa and CLQ as measures of inter-rater reliability of environmental ratings.

The focus in reliability studies in spatial contexts is often on test-retest reliability and internal consistency $[5,24]$, whereas the reliability across individuals rating the same environment has received much less attention. In the present study we develop the usage of both kappa and CLQ further for assessment of inter-rater reliability. We also explore how these measures can be used to investigate basic measurement properties of the rating scales, appropriate for identification of thresholds for dichotomizations and for decisions about how to handle indefinite or missing ratings. Reliability analysis of perceived greenness in the neighborhood environment will serve as an empirical example throughout the text.

\section{Methods}

\section{Survey data on environmental ratings}

In an extensive cross-sectional public health survey conducted in 2008 in Scania, southern Sweden, $\mathrm{n}=27$ 967 participants with a valid residential address rated the Scania Green Score, which covers five different aspects of the close natural outdoor environment within 5-10 minutes walking distance from home: serenity, wildness, species richness, spaciousness and cultural history (see Additional file 1 for phrasing of the questions) $[1,14]$. Each of these five items was rated on a 4graded ordinal scale: 1 = Disagree completely, 2 = Disagree, 3 =Agree, 4 =Agree completely. There was also a fifth option, 5 = Do not know/cannot say, and a sixth value, $6=$ Missing answer. We geocoded the residential address of each (index) participant and identified the first-order nearest neighbors within a specified maximum radius 500 meters in the main analyses. Each index participant and nearest neighbors within 500 meters were assumed to have rated overlapping neighborhood environments in the reliability analyses. Participants without a neighbor within 500 meters $(n=1757)$ were thus excluded. This left $n=26$ 210 sets of index participants matched with their nearest neighbors for analysis. The median distance to the nearest neighbor in this data set was 35 meters $(2.5$ - 97.5 percentiles $0-330$ meters). The distance to the nearest neighbor was generally longer in the semi-urban and rural areas of the study region. As a sensitivity analysis, we also evaluated three other maximum radiuses when identifying the firstorder nearest neighbors: 50 meters $(\mathrm{n}=15570), 100$ meters $(\mathrm{n}=21230)$ and 1000 meters $(\mathrm{n}=27364$ matched sets included in the analysis). In most cases, participants had a single nearest neighbor, but sometimes more than one participant was located at the same nearest neighbor distance. This typically occurred if participants lived at the same location (e.g., lived in the same multistory building), but it could also occur if residences of different neighboring participants happened to be the same distance away from the residence of the index participant.

\section{Framework for reliability analysis}

We consider a general setting with environmental ratings on a scale with $c$ categories, possibly including indefinite and missing answers as separate categories. We assess the agreement between environmental ratings of each matched set $i(\mathrm{i}=1,2,3, \ldots, n)$ of one index participant and its $m_{i}$ first-order nearest neighbors. The overall proportion of 
ratings across all index participants in category $j(j=1$, $2,3, \ldots, c)$ is $p_{j}$ such that $\sum_{j=1}^{c} p_{j}=1$. Let $x_{i}(j, k)$ denote the number of ratings in set $i$ for index rating $j$ and nearest neighbor rating $k$. The observed agreement proportion between a specific index rating $j=a$ and the nearest neighbor rating $k=b$ can be calculated across all sets of ratings as

$$
o_{a b}=\frac{1}{n} \sum_{i=1}^{n} \frac{1}{m_{i}} x_{i}(j=a, k=b),
$$

where $1 / m_{i}$ is used as a statistical weight to allow for inclusion of all first-order nearest neighbors if more than one neighbor is equally distant from the index participant [21]. Thus, after weighting, each match set will effectively contribute as though they contained two raters (the index rater and one nearest neighbor).

The kappa statistic [18] can be calculated specifically for the reliability of the same categorical rating $a$ as

$$
\kappa(a, a)=\frac{P_{O}(a, a)-P_{E}(a, a)}{1-P_{E}(a, a)},
$$

where the overall observed agreement proportion between the index participants and the nearest neighbors is

$$
P_{O}(a, a)=o_{a a}+\sum_{j \neq a} \sum_{k \neq a} o_{j k}=1-\sum_{j \neq a} o_{j a}-\sum_{k \neq a} o_{a k} .
$$

We assume that the nearest neighbors have the same overall distribution of ratings across categories as the index participants to calculate the chance-expected indexneighbor agreement under the null hypothesis of no agreement as

$$
P_{E}(a, a)=p_{a}^{2}+\left(1-p_{a}\right)^{2} .
$$

The calculation of kappa for the specific rating $a$ in Equation 2 is equivalent to first dichotomizing the rating scale as $a$ vs. $\neg a$ ("not $a$ ") and then calculating the overall kappa of the resulting $2 \times 2$-table (Figure 1 ).

\section{Calculation and interpretation of kappa}

Kappa can also be extended to measure the association between different index-neighbor ratings $a$ and $b$

$$
\kappa(a, b)=\frac{P_{O}(a, b)-P_{E}(a, b)}{1-P_{E}(a, b)},
$$

where the observed agreement proportion is

$$
P_{O}(a, b)=o_{a b}+\sum_{j \neq a} \sum_{k \neq b} o_{j k}=1-\sum_{j \neq a} o_{j b}-\sum_{k \neq b} o_{a k}
$$

and the chance-expected agreement is $P_{E}(a, b)=p_{a} p_{b}+$ $\left(1-p_{a}\right)\left(1-p_{b}\right)$ (Figure 2).

In words, kappa reflects how much the observed agreement exceeds chance-expected agreement, $P_{O}(a, b)-P_{E}$ $(a, b)$, expressed as a fraction or as a percentage of the maximum excess agreement, $1-P_{E}(a, b)$, that is possible to obtain. A kappa value of zero would imply no more than chance-expected agreement. When kappa is positive, observed agreement exceeds the chance-expected agreement (positive agreement). When kappa is negative, observed agreement is lower than chance-expected (negative agreement).

\section{Calculation and interpretation of CLQ}

We modify the original formulation of the CLQ [21] for the association between rating $a$ and $b$ to allow for sampling with replacement, i.e. the index rating is included in the calculation of the chance-expected agreement. As such, the CLQ can be calculated as:

$$
C L Q(a, b)=\frac{o_{a b}}{p_{a} \cdot p_{b}}
$$

where $o_{a b}$ is the observed agreement proportion (colocation) between index rating $a$ and nearest neighbor rating $b$ as defined in Equation 1 and $p_{a} \cdot p_{b}$ is the chanceexpected colocation. CLQ values range from 0 to a theoretical maximum that is dependent on the relative counts $p_{a}$ and $p_{b}$ as well as on certain geometrical constraints [21]. To get the theoretical maximum of CLQ we condition on the total proportion of ratings $p_{a}+p_{b}$ in category $a$ and $b$. By assuming equal occurrence of $a$ and $\mathrm{b}, \frac{p_{a}+p_{b}}{2}$, the maximum of CLQ can be obtained as

$$
C L Q_{\text {Max }}(a, b)=\frac{\left(p_{a}+p_{b}\right) / 2}{p_{a} \cdot p_{b}}=\frac{\left(p_{a}+p_{b}\right)}{2 \cdot p_{a} \cdot p_{b}} .
$$

A CLQ value of one would imply no more than chanceexpected agreement. For evidence of spatial association (positive agreement) between $a$ and $b$, we would want to see CLQ values above 1, i.e. a higher number of nearest neighbors with $b$ ratings than expected given its relative count in the population. When CLQ is below one, the observed colocation between $a$ and $b$ is less than expected (negative agreement). The lowest possible value of CLQ is zero, which only occurs if no nearest neighbor of the $a$ ratings has rated the environment as $b$. Note that both kappa and CLQ are asymmetric measures, i.e. $C L Q(a, b)$ is not necessarily equal to $C L Q(b, a)$.

\section{Comparisons of applicability of kappa and CLQ}

To facilitate comparison between kappa and CLQ, we can use that the observed agreement proportion $P_{O}(a, b)$ 


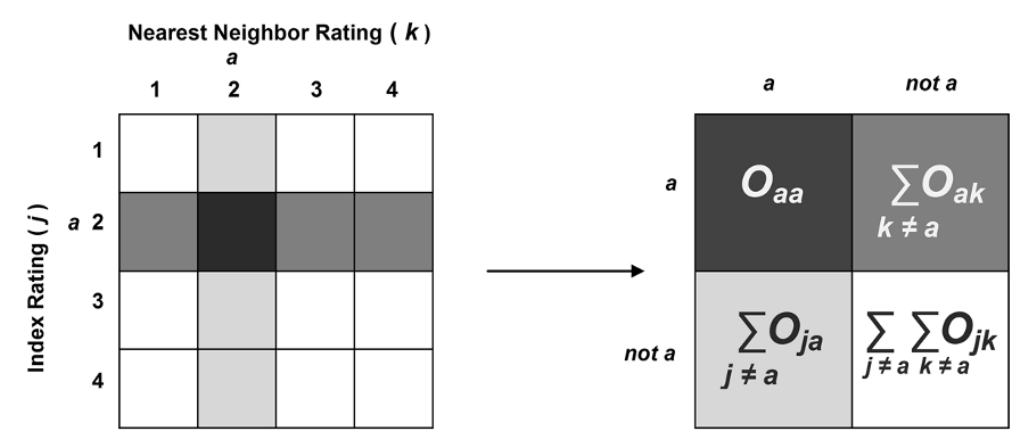

Figure 1 Converting the matrix of individual by nearest neighbor ratings to measure association for a specific rating. For a specific rating $a$ on a 4-category scale, all individual ratings are collapsed into a $2 \times 2$ table of a/not a ratings. Kappa is calculated from the compressed table.

between category $a$ and $b$ can be approximated by the following formula

$$
P_{O}(a, b) \approx 1-\left(p_{a}-o_{a b}\right)-\left(p_{b}-o_{a b}\right)
$$

(identity holds when the nearest neighbors and index participants have identical overall relative counts across category $a$ and $b$, i.e. when $\sum o_{j b}=p_{b}$ and $\left.\sum o_{a k}=p_{a}\right)$. Using the expressions for CLQ (Equation 4) and $C L Q_{\operatorname{Max}}$ (Equation 5), kappa can be approximated as

$$
\begin{aligned}
\kappa(a, b)= & \frac{P_{O}(a, b)-P_{E}(a, b)}{1-P_{E}(a, b)} \\
& \approx \frac{1-\left(p_{a}-o_{a b}\right)-\left(p_{b}-o_{a b}\right)-\left(p_{a} p_{b}+\left(1-p_{a}\right)\left(1-p_{b}\right)\right)}{1-\left(p_{a} p_{b}+\left(1-p_{a}\right)\left(1-p_{b}\right)\right)}= \\
= & \frac{o_{a b}-p_{a} p_{b}}{\left(p_{a}+p_{b}\right) / 2-p_{a} p_{b}}=\frac{\frac{o_{a b}}{p_{a} \cdot p_{b}}-1}{\frac{\left(p_{a}+p_{b}\right)}{2 \cdot p_{a} \cdot p_{b}}-1}=\frac{C L Q(a, b)-1}{C L Q_{\text {Max }}(a, b)-1} .
\end{aligned}
$$

In words, we can always normalize CLQ to an approximate kappa by subtracting the CLQ with one and dividing with its theoretical maximum minus one. With positive agreement for a specific categorical rating $a$ the CLQ will reflect a multiple of agreement more than expected, whereas kappa in percent will reflect how large the excess agreement is in relation to the maximum excess in agreement that can be obtained.

We present the reliability results below by reporting the CLQ together with kappa in percent as 'CLQ (kappa \%)'. For associations between different categorical ratings $a$ and $b$, CLQ and kappa will yield information about which ratings (including indefinite or missing ratings) cluster $(\mathrm{CLQ}>1$; Kappa $>0)$ or separate $(\mathrm{CLQ}<1$; Kappa $<0)$ in space. CLQ and kappa can thus be used not only to assess inter-rater reliability but also for assessing other basic measurement properties of rating scales. The results can for example serve as a basis for decisions about collapsing categories on the rating scale and for decisions about how to handle indefinite or missing ratings. We used the following three criteria, based on CLQ or kappa or both measures, to identify tentatively pairs of categorical ratings that could be combined without loss of reliability: i) a spatial clustering (CLQ > 1; Kappa >0) between the two ratings; ii) a CLQ that was of the same magnitude as the lowest of the two corresponding same-category CLQs; and iii) an increase in the same-category kappa of the collapsed vs. original ratings.

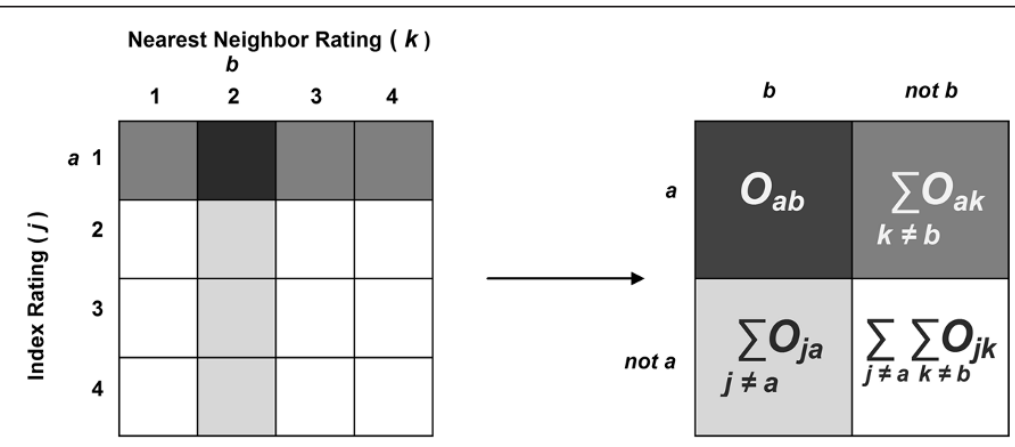

Figure 2 Converting the matrix of individual by nearest neighbor ratings to measure association for different ratings. For two different ratings $a$ and $b$ on a 4-category scale, all individual ratings are collapsed into a $2 \times 2$ table of $a /$ not $a$ versus $b /$ not $b$ ratings. Kappa is calculated from the compressed table. 


\section{Statistical analysis}

All statistical analyses were performed in $\mathrm{R}$ version 3.0.1 [25]. The distance calculations used in order to identify nearest neighbors were done with the fields package for spatial data version 6.9.1, function rdist [26]. We used the approximate standard error (SE) expression derived by Fleiss and Cohen for kappa between two raters [27] to assess tentatively the empirical evidence for more than chance-expected agreement (positive or negative) between category $a$ and $b$ :

$$
\begin{aligned}
\operatorname{SE}(\kappa(a, b)) & =\sqrt{\frac{P_{O}(a, b) \cdot\left(1-P_{O}(a, b)\right)}{n \cdot\left(1-P_{E}(a, b)\right)^{2}}} \\
& \approx \sqrt{\frac{\left(1-\left(p_{a}-o_{a b}\right)-\left(p_{b}-o_{a b}\right)\right) \cdot\left(\left(p_{a}-o_{a b}\right)+\left(p_{b}-o_{a b}\right)\right)}{n \cdot\left(1-p_{a} p_{b}-\left(1-p_{a}\right)\left(1-p_{b}\right)\right)^{2}}}
\end{aligned}
$$

\section{Results}

Reliability analysis was conducted for all five items of the Scania Green Score using the first-order nearest neighbors within the radius 500 meters in the main analyses. Results for three of the items, species richness, wildness and cultural history, showed similarities, whereas the results of the two remaining items, serenity and spaciousness, were different but similar to each other. We therefore focus on one item in each group, species richness and serenity, to illustrate how CLQ and kappa can be used when exploring inter-rater reliability and measurement properties (Tables 1, 2 and 3). Results for the three other items are presented in Additional file 2: Tables S1-S3. It should also be noted that both CLQ and kappa exhibited symmetry, i.e. similar associations between $a$ and $b$ as between $b$ and $a$, across all five items, although that need not always be the case with other spatial distributions of ratings. Due to the symmetry we will therefore limit the presentation in the contingency tables to results above the diagonals from top-left to bottom-right.

Among the index raters, $34 \%$ rated species richness in their neighborhood as $3=$ Agree and $12 \%$ rated species richness as $4=$ Agree completely (Table 1). There was clear evidence $(\mathrm{p}<0.001)$ of index-nearest neighbor reliability, i.e. observed agreement differing significantly from the chance-expected, for each of the informative ratings $1-4$ as shown in the top-left to bottom-right diagonal of Table 1 with CLQs ranging between 1.2 and 2.5. A CLQ of 2.5 as for rating 4 implies that the same rating among the nearest neighbors as among the index raters was 2.5 times as likely as could be expected by chance (observed agreement $3.4 \%$ vs. expected agreement 1.4\%; Table 1). These CLQ values correspond to $8.8 \%-20 \%$ of maximum agreement when expressed as kappa (Table 1 ). There was clear evidence $(\mathrm{p}<0.001)$ of positive agreement between the ratings $1=$ Disagree completely and
$2=$ Disagree $[$ CLQ $($ kappa $\%)=1.3(4.9 \%)]$ and between 3 and 4 [CLQ (kappa \%) $=1.3(6.6 \%)$ ], and these acrosscategories CLQs were of the same magnitude as the lowest of the corresponding same-category CLQs. On the other hand, the agreements between 1-3, 1-4, 2-3 and 2-4 were all negative with CLQs below one (CLQ range $0.33-0.84 ; \mathrm{p}<0.001)$. Taken together, these results suggest a spatial association between 1-2 (Disagree completelyDisagree) and between 3-4 (Agree-Agree completely) and that an appropriate threshold for dichotomization among the informative ratings $1-4$ would be between category 2 and 3.

The proportion of indefinite (5) or missing ratings (6) on species richness was $11.2 \%(7.0 \%+4.2 \%)$ among the index raters (Table 1). Indefinite and missing ratings did not seem to be distributed randomly (CLQ with the same rating was 1.4 and 1.5 , respectively; Table 1 ), although the statistical support for these associations was not consistently strong ( $\mathrm{p}=0.02$ for rating 5 and $\mathrm{p}=0.23$ for rating 6 ). The CLQ between 5 and 6 was somewhat lower (1.2) than the two corresponding same-category CLQs and statistically uncertain $(p>0.30)$. Spatial separation between ratings 5-6 and 3-4 with CLQs consistently below one was noted, but generally with substantial statistical imprecision inherent. Thus, there was some, but statistically weak, evidence suggesting that collapsing ratings 5 and 6 could be appropriate.

The consequences of collapsing ratings $1-2,3-4$ and 5-6 are presented in Table 2 . The inter-rater reliability of the collapsed ratings 1-2 (Disagree completely/Disagree) and 3-4. (Agree/Agree completely) expressed as kappa increased from $9.5 \% / 11 \%$ to $22 \%$ for ratings $1-2$ and from $8.8 \% / 20 \%$ to $26 \%$ for ratings $3-4$. Furthermore, the collapsed ratings 1-2 and 3-4 were clearly separated [CLQ $($ kappa \% $)=0.71(-23 \%) ; \mathrm{p}<0.001]$, providing additional support for combining the ratings in this way. Collapsing ratings 5-6 (Cannot say/Not answered) increased kappa from $3.8 \% / 2.5 \%$ to $4.9 \%$ ( $\mathrm{p}<0.001$ for kappa within ratings 5-6). Ratings 5-6 were more clearly separated from ratings $3-4$ when combined [CLQ (kappa \%) $=0.84(-3.3 \%)$; $\mathrm{p}<0.001$, but showed only weak spatial association with the collapsed ratings $1-2$ [CLQ (kappa \%) $=1.1(1.5 \%)$, $\mathrm{p}=0.03]$. Thus, a further grouping of ratings $5-6$ with ratings $3-4$ would be clearly inappropriate. On the other hand, there is no strong support for combining ratings $5-6$ with ratings $1-2$.

The serenity of the neighborhood environment was rated as $4=$ Agree completely by $28 \%$ and as $3=$ Agree by $43 \%$ of all index raters (Table 3). Index-nearest neighbor agreement was suggested for each of the informative ratings $1-4$ as shown in the diagonal of Table 3 with CLQs ranging between 1.1 and 1.9, corresponding to $4.6-23 \%$ of maximum agreement when expressed as kappa. There was positive agreement between rating 1 and 2 [CLQ 
Table 1 Index - nearest neighbor rater agreement on species richness in the close outdoor environment

\begin{tabular}{|c|c|c|c|c|c|c|c|c|c|c|c|c|c|}
\hline & \multirow{3}{*}{$\begin{array}{c}\text { Rel } \\
\text { freq }^{\mathrm{a}} \\
(\%)\end{array}$} & \multicolumn{12}{|c|}{ Nearest neighbor rater } \\
\hline & & \multicolumn{2}{|c|}{ 1. Disagree completely } & \multicolumn{2}{|c|}{ 2. Disagree } & \multicolumn{2}{|c|}{ 3. Agree } & \multicolumn{2}{|c|}{ 4. Agree completely } & \multicolumn{2}{|c|}{ 5. Cannot say } & \multicolumn{2}{|c|}{ 6. Not answered } \\
\hline & & Obs/Exp ${ }^{b}$ & CLQ (K \%) & Obs/Exp ${ }^{b}$ & $\overline{C L Q}(\mathrm{~K} \%)$ & Obs/Exp ${ }^{b}$ & $\overline{\text { CLQ (K \%) }}$ & Obs/Exp ${ }^{b}$ & $\overline{\text { CLQ (K \%) }}$ & Obs/Exp ${ }^{b}$ & $\overline{C L Q}(\mathrm{~K} \%)$ & Obs/Exp ${ }^{b}$ & CLQ (K \%) \\
\hline \multicolumn{14}{|l|}{ Index rater } \\
\hline 1 Disagree completely & 13 & $2.7 / 1.6$ & $1.7(9.5)^{* * *}$ & $4.9 / 3.9$ & $1.3(4.9)^{* * *}$ & $3.1 / 4.3$ & $0.72(-6.2)^{* * *}$ & $0.50 / 1.3$ & $0.33(-8.8)^{* * *}$ & $0.95 / 0.90$ & $1.1(1.0)$ & $0.62 / 0.54$ & $1.2(1.3)$ \\
\hline 2 Disagree & 31 & & & $12 / 9.3$ & $1.3(11)^{* * *}$ & $8.6 / 10$ & $0.84(-7.3)^{* * *}$ & $1.7 / 3.6$ & $0.47(-11)^{* * *}$ & $2.3 / 2.1$ & $1.1(1.2)$ & $1.3 / 1.3$ & $0.99(0.0)$ \\
\hline 3 Agree & 34 & & & & & $13 / 11$ & $1.2(8.8)^{* * *}$ & $5.1 / 4.0$ & $1.3(6.6)^{* * *}$ & $2.1 / 2.4$ & $0.88(-1.3)$ & $1.3 / 1.4$ & $0.90(-0.7)$ \\
\hline 4 Agree completely & 12 & & & & & & & $3.4 / 1.4$ & $2.5(20)^{* * *}$ & $0.52 / 0.83$ & $0.63(-3.1)^{*}$ & $0.40 / 0.50$ & $0.79(-1.1)$ \\
\hline 5 Cannot say & 7.0 & & & & & & & & & $0.70 / 0.49$ & $1.4(3.8)^{*}$ & $0.36 / 0.29$ & $1.2(1.6)$ \\
\hline 6 Not answered & 4.2 & & & & & & & & & & & $0.26 / 0.18$ & $1.5(2.5)$ \\
\hline
\end{tabular}

${ }^{*} 0.01 \leq \mathrm{p}<0.05 ;{ }^{* * *} \mathrm{p}<0.001$.

a Relative frequency of index ratings in each category

bobserved/Expected relative frequency (\%) used in the calculation of the colocation quotient (CLQ). 
Table 2 Index - nearest neighbor rater agreement on species richness collapsed into a $3 \times 3$-table

\begin{tabular}{|c|c|c|c|c|c|c|c|}
\hline & \multirow{3}{*}{$\begin{array}{c}\text { Rel } \\
\text { freq }^{\mathrm{a}} \\
(\%)\end{array}$} & \multicolumn{6}{|c|}{ Nearest neighbor rater } \\
\hline & & \multicolumn{2}{|c|}{ 1-2 Disagree completely/Disagree } & \multicolumn{2}{|c|}{ 3-4 Agree/Agree completely } & \multicolumn{2}{|c|}{ 5-6 Cannot say/Not answerec } \\
\hline & & Obs/Exp & CLQ (K \%) & Obs/Exp & CLQ (K \%) & Obs/Exp ${ }^{b}$ & CLQ (K \%) \\
\hline \multicolumn{8}{|l|}{ Index rater } \\
\hline 1-2 Disagree completely/Disagree & 43 & $24 / 19$ & $1.3(21)^{* * *}$ & $14 / 19$ & $0.71(-23)^{* * *}$ & $5.1 / 4.8$ & $1.1(1.5)^{*}$ \\
\hline 3-4 Agree/Agree completely & 46 & & & $27 / 21$ & $1.3(26)^{* * *}$ & $4.3 / 5.1$ & $0.84(-3.3)^{* * *}$ \\
\hline 5-6 Cannot say/Not answered & 11 & & & & & $1.7 / 1.3$ & $1.3(4.9)^{* * *}$ \\
\hline
\end{tabular}

(kappa \%) $=1.6(4.6 \%)] ; \mathrm{p}<0.001$ with the CLQ being compatible with the lowest of corresponding two samecategory CLQs (1.5), whereas a weak spatial separation between rating 3 and 4 was observed [CLQ (kappa \%) = $0.92(-3.7 \%), \mathrm{p}<0.001$; Table 3]. There was clear evidence of spatial separation between rating 1 and 4 [CLQ $($ kappa $\%)=0.49(-4.8 \%) ; \mathrm{p}<0.001]$ and between 2 and 4 [CLQ $($ kappa \%) = $0.51(-13 \%)$; $<0.001$ ], while no apparent association was detected between 1 and 3 or between 2 and 3 . These results thus suggest a spatial colocation of rating 1 and 2 while rating 3 and 4 seem to be separate entities. As for species richness, the spatial association with indefinite (5) and missing ratings (6) was somewhat lower (1.4) than the two corresponding same-category CLQs (1.7 and 1.6, respectively; Table 3 ) and statistically uncertain. A negative association between rating 4 and 5 was observed [CLQ (kappa \%) $=0.57(-2.9 \%), \mathrm{p}=0.002$ ]

The consequences of collapsing ratings $1-2$ and 5-6 are presented in Table 4. The inter-rater reliability of the collapsed ratings 1-2 (Disagree completely/Disagree) and 5-6 (Cannot say/Not answered) expressed as kappa increased from $5.9 \% / 11 \%$ to $17 \%$ for ratings $1-2$ and from $2.2 \% / 3.0 \%$ to $4.4 \%$ for ratings $5-6$. By contrast, collapsing ratings 3-4 (Agree/Agree completely) would not increase the reliability: kappa $=19 \%$ for ratings $3-4$ combined (not in tables) vs. $4.6 \% / 23 \%$ for rating 3 and 4 as separate categories (Table 4).

The presented kappa values were calculated from the definition in Equation 3, but can also be approximated closely by normalizing the corresponding CLQ using Equation 6. As an example, the reported kappa between rating 3 and 4 for species richness was $0.066=6.6 \%$, which can also be obtained approximately from CLQ and the relative counts in Table 1 as

$$
\begin{aligned}
\kappa(3,4) & \approx \frac{C L Q(3,4)-1}{C L Q_{\operatorname{Max}}(3,4)-1}=\frac{C L Q(3,4)-1}{\frac{\left(p_{3}+p_{4}\right)}{2 \cdot p_{3} \cdot p_{4}}-1} \approx \frac{1.3-1}{\frac{(0.337+0.118)}{2 \cdot 0.337 \cdot 0.118}-1} \\
& \approx 0.064=6.4 \%
\end{aligned}
$$

In the sensitivity analysis, an increased maximum radius when identifying the first-order nearest neighbors was associated with the inclusion of more rural environments and thereby larger proportions of positive assessments of neighborhood greenness (not in tables). If anything, the reliability increased somewhat with larger radiuses. As an example, the kappa for species richness, rating 4 , increased from $18 \%$ with radius 50 meter $(8.5 \%$ of all included index ratings) to $22 \%$ with radius 1000 meter $(13.1 \%$ of all included index ratings). It thus seems that the neighbor environments in the rural areas were easier to agree on, despite less spatial overlap (i.e. larger distance to the nearest neighbors) for those environments.

\section{Discussion}

Our empirical example demonstrates the usefulness of kappa and CLQ as tools for assessing reliability and measurement properties of environmental rating scales when used at geographical point locations. The two measures are interchangeable, i.e. kappa can be accurately approximated from CLQ and vice versa, but can as seen in our example be used for somewhat different purposes in the reliability analysis. CLQ is a measure of spatial association and was used to identify sets of ratings with compatible colocation across as within categories, whereas kappa is a measure of agreement and was used to assess effects on reliability when clustered sets of ratings were combined. An advantage of kappa is that it is a well-known measure of agreement in other (non-spatial) settings within epidemiology and clinical research. The present study broadens the usage of kappa to studies of reliability and of associations between different categorical ratings in spatial contexts. By contrast, the novel CLQ is an explicit spatial measure that requires information on the spatial arrangement of ratings. CLQ is designated to show the level of spatial association among each pair of ratings and has its strength in the appealing interpretation of how many more times more likely concordant ratings and observed colocations are than chance. When there is interest in exploring the spatial structure of agreement in environmental ratings, CLQ may be the preferred approach.

Inter-rater reliability was in the present study demonstrated for all five items of the Scania Green Score, albeit with clear differences across the items. Previous 
Table 3 Index - nearest neighbor rater agreement on serenity in the close outdoor environment

\begin{tabular}{|c|c|c|c|c|c|c|c|c|c|c|c|c|c|}
\hline & \multirow{3}{*}{$\begin{array}{c}\text { Rel } \\
\text { freq }^{\mathrm{a}} \\
(\%)\end{array}$} & \multicolumn{12}{|c|}{ Nearest neighbor rater } \\
\hline & & \multicolumn{2}{|c|}{ 1. Disagree completely } & \multicolumn{2}{|c|}{ 2. Disagree } & \multicolumn{2}{|c|}{ 3. Agree } & \multicolumn{2}{|c|}{ 4. Agree completely } & \multicolumn{2}{|c|}{ 5. Cannot say } & \multicolumn{2}{|c|}{ 6. Not answered } \\
\hline & & Obs/Exp ${ }^{b}$ & CLQ (K \%) & Obs/Exp ${ }^{b}$ & CLQ (K \%) & Obs/Exp ${ }^{b}$ & CLQ (K \%) & Obs/Exp ${ }^{b}$ & CLQ (K \%) & Obs/Exp ${ }^{b}$ & CLQ (K \%) & Obs/Exp ${ }^{b}$ & CLQ (K \%) \\
\hline \multicolumn{14}{|l|}{ Index rater } \\
\hline 1 Disagree completely & 5.7 & $0.60 / 0.32$ & $1.9(5.9)^{* * *}$ & $1.6 / 10$ & $1.6(4.6)^{* * *}$ & $2.2 / 2.4$ & $0.91(-1.1)$ & $0.77 / 1.6$ & $0.49(-4.8)^{* * *}$ & $0.29 / 0.20$ & $1.5(1.9)$ & $0.25 / 0.20$ & $1.3(1.7)$ \\
\hline 2 Disagree & 18 & & & $4.8 / 3.1$ & $1.5(11)^{* * *}$ & 7.3/7.3 & $0.99(-0.2)$ & $2.5 / 4.9$ & $0.51(-13)^{* * *}$ & 0.80/0.62 & $1.3(1.7)$ & $0.67 / 0.62$ & $1.1(0.7)$ \\
\hline 3 Agree & 42 & & & & & 19/17 & $1.1(4.6)^{* * *}$ & $11 / 12$ & $0.92(-3.7)^{* * *}$ & $1.5 / 1.5$ & $1.0(-0.0)$ & $1.4 / 1.5$ & $0.95(-0.2)$ \\
\hline 4 Agree completely & 28 & & & & & & & $12 / 7.8$ & $1.6(23)^{* * *}$ & $0.56 / 0.98$ & $0.57(-2.9)^{* *}$ & $0.79 / 0.98$ & $0.80(-1.2)$ \\
\hline 5 Cannot say & 3.5 & & & & & & & & & $0.21 / 0.12$ & $1.7(2.2)$ & $0.17 / 0.12$ & $1.4(2.2)$ \\
\hline 6 Not answered & 3.5 & & & & & & & & & & & $0.20 / 0.12$ & $1.6(3.0)$ \\
\hline
\end{tabular}

${ }^{* *} \mathrm{p}<0.01 ; * * \mathrm{p}<0.001$.

a Relative frequency of index ratings in each category.

${ }^{\mathrm{b}}$ Observed/Expected relative frequency (\%) used in the calculation of the colocation quotient (CLQ). 
Table 4 Index - nearest neighbor rater agreement on serenity in the close outdoor environment

\begin{tabular}{|c|c|c|c|c|c|c|c|c|c|}
\hline & \multirow{3}{*}{$\begin{array}{c}\text { Rel } \\
\text { freq }^{\mathrm{a}} \\
(\%)\end{array}$} & \multicolumn{8}{|c|}{ Nearest neighbor rater } \\
\hline & & \multicolumn{2}{|c|}{$\begin{array}{c}1-2 . \text { Disagree } \\
\text { completely/Disagree }\end{array}$} & \multicolumn{2}{|c|}{ 3. Agree } & \multicolumn{2}{|c|}{ 4. Agree completely } & \multicolumn{2}{|c|}{$\begin{array}{l}5 \text { - 6. Cannot say/ } \\
\text { Not answered }\end{array}$} \\
\hline & & Obs/Exp ${ }^{b}$ & $\overline{C L Q}(\mathrm{~K} \%)$ & Obs/Exp ${ }^{b}$ & CLQ (K \%) & Obs/Exp ${ }^{b}$ & CLQ (K \%) & Obs/Exp ${ }^{b}$ & CLQ (K \%) \\
\hline \multicolumn{10}{|l|}{ Index rater } \\
\hline 1-2 Disagree completely/Disagree & 23 & $8.5 / 5.4$ & $1.6(17)^{* * *}$ & $9.5 / 9.7$ & $0.97(-1.2)$ & $3.3 / 6.5$ & $0.51(-16)^{* * *}$ & $2.0 / 1.6$ & $1.2(2.9)^{* *}$ \\
\hline 3 Agree & 42 & & & 19/17 & $1.1(4.6)^{* * *}$ & $11 / 12$ & $0.92(-3.7)^{* * *}$ & $2.9 / 2.9$ & $0.98(-0.2)$ \\
\hline 4 Agree completely & 28 & & & & & $12 / 7.8$ & $1.6(23)^{* * *}$ & $1.4 / 2.0$ & $0.68(-3.9)^{* *}$ \\
\hline 5-6 Cannot say/Not answered & 7.0 & & & & & & & $0.77 / 0.49$ & $1.6(4.4)^{* *}$ \\
\hline
\end{tabular}

**p $<0.01 ; * * * 0<0.001$.

${ }^{\text {a }}$ Relative frequency of index ratings in each category.

${ }^{b}$ Observed/Expected relative frequency (\%) used in the calculation of the colocation quotient (CLQ).

studies using these items have dichotomized the scale as Disagree completely or Disagree vs. Agree or Agree completely $[1,14]$. The present results provide support for treating the scale in this way only for three of the items, species richness, wildness and cultural history. By contrast, the analysis of the two other items, serenity and spaciousness, provided no firm support for dichotomizations. Furthermore, indefinite and missing answers have in previous studies been combined with Disagree completely/Disagree [1,14], but the present reliability analysis only showed a weak spatial association that would motivate this. It should be noted that we restricted the attention to reliability issues when different groupings of the original ratings were considered. In practice, validity issues should also be addressed, e.g. environmental ratings that reflect different underlying constructs might be inappropriate to collapse even if they exhibit colocation. The consequence of inappropriate collapsing of environmental ratings would be equivalent to non-differential misclassification of exposure. Such misclassification yields bias towards the null if the final classification used in the epidemiological analysis is dichotomous, whereas the resulting bias is less predictable in case of a polychotomous classification [28].

The large survey sample, with 26210 sets of index participants matched with their nearest neighbors, made it possible to conduct detailed reliability and association analyses with sufficient statistical precision for the informative ratings $1-4$, whereas the statistical power to detect spatial associations with the less prevalent indefinite and missing ratings was lower. Another study limitation was that the index-neighbor ratings did not refer to the exactly the same neighborhood environment. Although the distance between the residences within the matched sets of raters was generally short, differences in the geographical point locations probably contributed to dispersion in the ratings. When designing reliability studies of environmental ratings, it is essential that the geographical sampling is such that sufficient numbers of participants are rating the same environments.
Kappa and CLQ differ with respect to their maximum values. Kappa is normalized and its maximum is therefore always $100 \%$ when expressed as a percentage. By contrast, CLQ is not normalized; the maximum value will be higher the lower the relative frequency of the least common of the two ratings is. Maximum CLQ will also be higher the more similar the relative frequencies of the two ratings are. Thus, it is not obvious to judge how impressive a CLQ of, say, 1.5, is, unless the corresponding kappa value is also presented. In our empirical example, the kappa ranged between $2 \%$ and $11 \%$ for different CLQs of 1.5. For this reason, we used the change in kappa rather than in CLQ to assess the effect on reliability when rating categories were combined. For a given agreement proportion, both kappa and CLQ will be dependent on the number of categories and marginal frequencies of the evaluated contingency table. This is not necessarily a weakness of the measures per se, since identical agreement proportions should be judged differently depending on the chance-expected agreement [29]. Thus, when comparing reliability and spatial associations across study settings, the concern should not be differences in marginal frequencies as such, but rather differences in the proportion of local environments that are difficult to decide on [29].

A concern with the kappa measure, which also applies for the CLQ, is that it does not distinguish between systematic and random disagreement [30]. However, this was less of a problem in our empirical example where index and nearest neighbor had similar overall distributions of ratings, suggesting that the disagreement is random rather than systematic. The reported kappa values (at most $10-21 \%$ when calculated between the same informative ratings $1-4$ of an item) may seem low compared with usual requirements for sufficient inter-rater agreement in other settings. An important explanation for the low agreement, besides differences in the geographical point locations, is likely to be factors on the individuallevel related to socioeconomy, e.g. housing situation, country of origin and education, which have been shown 
to influence the perception of the neighborhood [31]. The influence of such individual factors may bias epidemiological associations between individual ratings of the environment and health outcomes if not adjusted for [14]. One way of improving the generalizability of the individual ratings, as well as limiting the bias, would be to aggregate all ratings within a neighborhood $[9,14,24]$. However, the results of the present study imply that such aggregations should not be conducted without firstly analyzing reliability and measurement properties of the ratings at the individual-level. Further matching for factors known to influence perception of the neighborhood could be considered in reliability analyses but would limit generalizability of the results and, at least in the present study, decrease sample size.

The present study only assessed reliability and colocation globally, i.e. only for the entire survey cohort, and thereby ignoring much of the spatial context. For simplicity reasons, all matched sets obtained equal statistical weight in the analyses, regardless of the number of and distance to nearest neighbors. The CLQ was recently extended to a local counterpart using principles of geographical weighting based on the distance between the objects [32]. A natural extension of the present study would therefore be to extend the analysis spatially, aiming at identifying individual-level or local geographical determinants of the reliability and colocation. We only assessed the statistical uncertainty of the suggested spatial associations tentatively; developing and validating formulas for the standard error is an issue that also deserves further attention.

In conclusion, both the extended definition of the wellknown kappa statistic and the novel CLQ were shown to be appropriate tools for investigating inter-rater reliability and spatial associations between environmental assessments on categorical ratings scales. Such reliability analyses should serve as guidance for subsequent epidemiological studies of associations between environmental ratings, health and wellbeing.

\section{Additional files}

Additional file 1: Survey questions in Scania Green Score.

Additional file 2: Table S1. Index - nearest neighbor rater agreement on wildness in the close outdoor environment. Table S2. Index - nearest neighbor rater agreement on spaciousness in the close outdoor environment. Table S3. Index - nearest neighbor rater agreement on cultural history in the close outdoor environment.

Abbreviation

CLQ: Colocation quotient.

\section{Competing interests}

The authors declare that they have no competing interest.

\section{Authors' contributions}

All authors have participated in the initiation, planning, execution, and analysis of this study. RR was responsible for the statistical programming with assistance from EC. JB was responsible for writing the manuscript with assistance from RR and EC. All authors have read, revised and approved the final version submitted.

\section{Acknowledgement}

The work was funded by Swedish Research Council (SIMSAM Lund) and by FORTE (Swedish Research Council for Health, Working Life and Welfare.

\section{Author details}

${ }^{1}$ Division of Occupational and Environmental Medicine, Lund University, SE-221 85 Lund, Sweden. ${ }^{2}$ Department of Community Medicine and Health Care, University of Connecticut School of Medicine, 263 Farmington Avenue, Farmington, CT 06030-6325, USA.

Received: 26 June 2014 Accepted: 14 October 2014

Published: 23 October 2014

\section{References}

1. de Jong K, Albin M, Skärbäck E, Grahn P, Björk J: Perceived green qualities were associated with neighborhood satisfaction, physical activity, and general health: results from a cross-sectional study in suburban and rura Scania, southern Sweden. Health Place 2012, 18(6):1374-1380.

2. Grahn P, Stigsdotter UK: The relation between perceived sensory dimensions of urban green space and stress restoration. Landsc Urban Plan 2010, 94(3-4):264-275.

3. Korpela KM, Ylen M, Tyrvainen L, Silvennoinen $H$ : Favorite green, waterside and urban environments, restorative experiences and perceived health in Finland. Health Promot Int 2010, 25(2):200-209.

4. Gidlöf-Gunnarsson A, Öhrström E: Noise and well-being in urban residential environments: the potential role of perceived availability to nearby green areas. Landsc Urban Plan 2007, 83(2-3):115-126.

5. Brownson RC, Hoehner CM, Day K, Forsyth A, Sallis JF: Measuring the built environment for physical activity: state of the science. Am J Prev Med 2009, 36(4 Suppl):S99-123 e112.

6. Prins RG, Oenema A, van der Horst K, Brug J: Objective and perceived availability of physical activity opportunities: differences in associations with physical activity behavior among urban adolescents. Int J Behav Nutr Phys Act 2009, 6:70.

7. Auchincloss AH, Diez Roux AV, Mujahid MS, Shen M, Bertoni AG, Carnethon MR: Neighborhood resources for physical activity and healthy foods and incidence of type 2 diabetes mellitus: the Multi-Ethnic study of Atherosclerosis. Arch Intern Med 2009, 169(18):1698-1704.

8. Babisch W, Pershagen G, Selander J, Houthuijs D, Breugelmans O, Cadum E, Vigna-Taglianti F, Katsouyanni K, Haralabidis AS, Dimakopoulou K, Sourtzi P, Floud S, Hansell AL: Noise annoyance-a modifier of the association between noise level and cardiovascular health? Sci Total Environ 2013, 452-453:50-57.

9. Oglesby L, Kunzli N, Monn C, Schindler C, Ackermann-Liebrich U, Leuenberger $P$ : Validity of annoyance scores for estimation of long term air pollution exposure in epidemiologic studies: the Swiss Study on Air Pollution and Lung Diseases in Adults (SAPALDIA). Am J Epidemiol 2000, 152(1):75-83.

10. Deguen $\mathrm{S}$, Segala $\mathrm{C}$, Pedrono G, Mesbah M: A new air quality perception scale for global assessment of air pollution health effects. Risk Anal 2012, 32(12):2043-2054.

11. Gebel K, Bauman AE, Sugiyama T, Owen N: Mismatch between perceived and objectively assessed neighborhood walkability attributes: Prospective relationships with walking and weight gain. Health Place 2011, 17:519-524.

12. Van Dyck D, Veitch J, De Bourdeaudhuij I, Thornton L, Ball K: Environmental perceptions as mediators of the relationship between the objective built environment and walking among socio-economically disadvantaged women. Int J Behav Nutr Phys Act 2013, 10:108.

13. Leslie E, Sugiyama T, lerodiaconou D, Kremer P: Perceived and objectively measured greeness of neighborhoods: are they measuring the same thing? Landsc Urban Plan 2010, 95:28-33.

14. de Jong K, Albin M, Skärbäck E, Grahn P, Wadbro J, Merlo J, Björk J: Area-aggregated assessments of perceived environmental attributes 
may overcome single-source bias in studies of green environments and health: results from a cross-sectional survey in southern Sweden. Environ Health 2011, 10(1):4.

15. McCormack GR, Cerin E, Leslie E, du Toit L, Owen N: Objective versus perceived walking distances to destinations: Correspondence and predictive validity. Environ Behav 2008, 40(3):401-425.

16. Forsberg B, Stjernberg N, Wall S: People can detect poor air quality well below guideline concentrations: a prevalence study of annoyance reactions and air pollution from traffic. Occup Environ Med 1997, 54(1):44-48.

17. Cohen J: A coefficient of agreement for nominal scales. Educ Psychol Meas 1960, 20(1):37-46.

18. Fleiss $\mathrm{JL}$ : Measuring nominal scale agreement among many raters. Psychol Bull 1971, 76(5):378-382.

19. Kvalseth TO: Weighted specific-category kappa measure of interobserver agreement. Psychol Rep 2003, 93(3 Pt 2):1283-1290.

20. Cohen J: Weighted kappa: nominal scale agreement with provision for scaled disagreement or partial credit. Psychol Bull 1968, 70(4):213-220.

21. Leslie TF, Kronenfeld BJ: The colocation quotient: a new measure of spatial association between categorical subsets of points. Geogr Anal 2011, 43(3):306-326.

22. Djira GD, Schaarschmidt F, Bichaka F: Inferences for selected location quotients with applications to health outcomes. Geogr Anal 2010, 42(3):288-300.

23. Ceyhan E: Overall and pairwise segregation tests based on nearest neighbor contingency tables. Comput Stat Data An 2009, 53(8):2786-2808.

24. Mujahid MS, Diez Roux AV, Morenoff JD, Raghunathan T: Assessing the measurement properties of neighborhood scales: from psychometrics to ecometrics. Am J Epidemiol 2007, 165(8):858-867.

25. The R project for statistical computing. [www.r-project.org]

26. Package 'fields'. [http://cran.r-project.org/web/packages/fields/fields.pdf]

27. Fleiss JL, Cohen J, Everitt BS: Large sample standard errors of kappa and weighted kappa. Psychol Bull 1969, 72(5):323-327.

28. Dosemeci M, Wacholder S, Lubin JH: Does nondifferential misclassification of exposure always bias a true effect toward the null value? Am J Epidemiol 1990, 132(4):746-748.

29. Vach W: The dependence of Cohen's kappa on the prevalence does not matter. J Clin Epidemiol 2005, 58(7):655-661.

30. Svensson E: Application of a rank-invariant method to evaluate reliability of ordered categorical assessments. J Epidemiol Biostatist 1998, 3(4):403-409.

31. Kamphuis CB, Mackenbach JP, Giskes K, Huisman M, Brug J, van Lenthe FJ: Why do poor people perceive poor neighbourhoods? The role of objective neighbourhood features and psychosocial factors. Health Place 2010, 16(4):744-754.

32. Cromley RG, Hanink DM, Bentley GC: Geographically weighted colocation quotients: specification and application. Prof Geographer 2014, 66(1):138-148

doi:10.1186/1476-069X-13-86

Cite this article as: Björk et al:: Exploring inter-rater reliability and measurement properties of environmental ratings using kappa and colocation quotients. Environmental Health 2014 13:86.

\section{Submit your next manuscript to BioMed Central and take full advantage of:}

- Convenient online submission

- Thorough peer review

- No space constraints or color figure charges

- Immediate publication on acceptance

- Inclusion in PubMed, CAS, Scopus and Google Scholar

- Research which is freely available for redistribution 